\title{
Uso de la urografía por resonancia magnética en el estudio del aparato urinario frente a la urografía convencional
}

\author{
Ramos de Campos M, Juan Escudero JU, Navalón Verdejo P, Ordoño Domínguez F, \\ Fabuel Deltoro M, Zaragoza Orts J.
}

\author{
Servicio de Urología. Consorcio Hospital General Universitario de Valencia
}

Actas Urol Esp 2007;31(3):253-261

\section{RESUMEN}

USO DE LA UROGRAFÍA POR RESONANCIA MAGNÉTICA EN EL ESTUDIO DEL APARATO URINARIO FRENTE A LA UROGRAFÍA CONVENCIONAL

La urografía convencional (UIV) es una exploración fundamental en la valoración del aparato urinario no exenta de morbilidad, como reacciones adversas al contraste (vasovagales y anafilácticas), neurotoxicidad, nefrotoxicidad, así como la irradiación ionizante a la que somete al paciente. Por este motivo se desarrollan técnicas de imagen alternativas como la urografia por resonancia magnética (RM) o urorresonancia.

Objetivos: Presentamos un estudio que valora la precisión diagnóstica, especificidad y sensibilidad de la urorresonancia y de la UIV como exploración morfológica y funcional del aparato urinario; así como de la calidad de las imágenes urográficas obtenidas con la RM frente a la UIV.

Material y métodos: 150 pacientes han sido sometidos a un estudio de RM, en 63 de ellos se realizó también estudio urográfico convencional, con apreciación de señales de alta intensidad en T2 correspondientes al fluido abdominal y retroperitoneal, empleando previamente furosemida a bajas dosis, y en un estudio final, administrando gadolinio a razón de 0,1 mg./Kg. La prueba fue indicada en pacientes con antecedentes de reacciones adversas al contraste yodado, fracaso renal agudo o crónico, anulación funcional renal, pacientes embarazadas y en edad infantil así como cuando la UIV no fue diagnóstica. Se estudió la capacidad de ambas pruebas para el diagnóstico de obstrucción urinaria y de la etiología de esta. También se evalúo la calidad de las imágenes que ofrece el estudio urográfico con RM.

Resultados: Se obtuvieron imágenes de alta resolución con la RM de toda la vía urinaria superior, especialmente de pelvis renal, no artefactadas por la peristalsis o la interposición del fluido intestinal. En el 83,3\% de los casos la exploración reveló patología urológica. La precisión diagnóstica de la causa de afectación del aparato urinario fue de un 83,3\%, con una sensibilidad del $89,6 \%$, una especificidad del $69,2 \%$, valor predictivo positivo del $86,6 \%$ y valor predictivo negativo del $75 \%$.

Conclusión: La urografia por RM es una técnica con una elevada sensibilidad para el estudio del aparato urinario, que puede ser empleada como alternativa a la urografia convencional especialmente en los casos de contraindicación de la radiación ionizante, alergia al contraste, así como en pacientes con fracaso renal, pero que ofrece un estudio morfológico y funcional más amplio, con elevada calidad de imágenes, pudiendo desplazar a los estudios convencionales en un plazo corto o medio de tiempo.

Palabras clave: Urorresonancia. Urografia intravenosa. Tracto urinario.

\section{ABSTRACT}

\section{USE OF UROGRAPHY BY MAGNETIC RESONANCE FOR THE STUDY OF THE URINARY APPARATUS VERSUS}

\section{CONVENTIONAL UROGRAPHY}

Conventional urography (IVU) is an essential examination for the assessment of urinary tract but it is not free of complications, such as adverse reactions to contrast agents used (vasovagal and anaphylactic reactions), neurotoxicity, nephrotoxicity, as well as the damage due to the ionizing irradiation applied to the patient. For this reason, alternative imaging techniques such as magnetic resonance (MR) urography or uro-resonance have been developed.

Objective: We present a case study assessing the diagnostic accuracy, specificity and sensitivity of uro-resonance and IVU as a morphological and functional examination of the urinary tract; and a quality study of the urographic images obtained with MR versus IVU.

Materials and methods: 150 patients have submited to a MR study, 63 of them with an IVU study already performed, acquiring high-intensity signals at T2 corresponding to abdominal and retroperitoneal fluid, initially using furosemide at low doses and, in a final study, administering gadolinium at a rate of $0.1 \mathrm{mg} / \mathrm{Kg}$. The test was indicated in patients with antecedents of adverse reactions to iodine contrast, acute or chronic kidney failure, functional cancellation of the kidneys, pregnant patients and those in paediatric age. The capacity of diagnosis of urinary obstruction and the aetiology of this obstruction of both tests was studied, as well as the quality of the images obtained by the urographic study using MR.

Results: High resolution images were obtained of all the upper urinary tracts using MR, especially in the renal pelvis, without artefacts caused by peristalsis or intestinal fluid interposition. In $83.3 \%$ of cases, examinations revealed urological pathology. The diagnostic accuracy of the involvement cause of the urinary tract was $83.3 \%$, with a sensitivity of $89.6 \%$, a specificity of $69.2 \%$, a positive predictive value of $86.6 \%$ and a negative predictive value of $75 \%$.

Conclusion: MR urography is a high sensitive technique for the study of urinary tract, used as an alternative to conventional urography particularly in cases of the contraindication of ionizing radiation or allergy to the contrast agent, as well as in patients with renal failure, and offers a wider morphological and functional study, with a high image quality, able to displace conventional examinations in the short or medium term.

Keywords: Magnetic Resonance Urography. Intravenous Urography. Urinary Tract . 
$\mathrm{E}$ n la valoración del aparato urinario, el urólogo dispone de múltiples técnicas de imagen, de ellas, la urografía intravenosa (UIV) representa la exploración principal, especialmente desde el desarrollo de contrastes no iónicos, permitiendo un estudio morfológico y funcional del aparato urinario, y sobre todo, del tracto superior. Sin embargo, el desarrollo de técnicas alternativas de imagen tales como la ecografía, la tomografía axial computerizada (TAC) y la resonancia magnética (RM), ha desplazado parcialmente a la UIV. Estas técnicas permiten una mejor valoración del retroperitoneo y del parénquima renal, además, tanto la ecografía como la RM, ni exponen a radiaciones ionizantes, ni precisan de contrastes yodados responsables de reacciones adversas angiotóxicas, neurotóxicas y nefrotóxicas y que además esta contraindicado su uso en pacientes con fracaso renal agudo o crónico, mieloma múltiple, diabetes y embarazadas ${ }^{1}$.

En los últimos años el desarrollo de nuevos hardwares y softwares en el terreno de la RM, ha permitido que esta técnica alcance una calidad de imagen comparable a la de la TAC ${ }^{2}$. En la actualidad es posible obtener imágenes urográficas con RM gracias a las secuencias altamente potenciadas en $\mathrm{T} 2$, capaces de obtenerse rápidamente con técnicas como el RARE (Rapid Acquisition with Relaxation Enhancement) y al HASTE ( Half-Fourier Acquisition Single-shot Turbo spin-Echo) ${ }^{3,4}$, pero también, podemos adquirir estas secuencias urográficas por RM de forma similar a la UIV convencional adquiriendo imágenes en fase excretora tras la administración de gadolinio, que realza la vía urinaria en secuencias T1 (Diuretic Contrast-Enhanced Magnetic Resonance Urography).

Una de las principales limitaciones de la RM es el prolongado tiempo necesario para realizar la exploración, que se ha reducido notablemente con el desarrollo de estos nuevos sistemas de adquisición, que permiten obtener secuencias urográficas en una sola apnea sin uso de contrastes. El RARE incrementa el número de ecos a 256 , lo que proporciona un largo tren de ecos de unos $1.200 \mathrm{mseg}$, altamente potenciados en T2. Con un solo disparo se alcanza un corte de un grosor entre $20-80 \mathrm{~mm}$, sin precisar un posterior procesamiento $^{5}$. El HASTE es una técnica que emplea múltiples disparos, pero sólo adquiere la mitad de las líneas de imagen para ganar tiempo, calculando por simetría la otra mitad. Permite adquirir de 10-20 cortes finos (de 3-5 mm) en una sola apnea y en un total de 15 a 20 segundos. Pese a no estar las imágenes tan potenciadas en T2 como en el RARE, el fluido estático sigue contrastando intensamente sobre el resto de señales de baja intensidad procedente de los tejidos circundantes $^{4,6}$.

La técnica HASTE puede ser complementada con un posterior procesamiento o proyección de máxima intensidad (MIP), que conserva las estructuras con alta intensidad de señal tras sumar los múltiples cortes finos, lo que permite rotar la imagen y obtener la proyección deseada, y es capaz de eliminar la superposición de otro fluido no procedente del aparato urinario. El resultado es una menor artefactación por el fluido intestinal, aunque el mismo procesamiento MIP puede introducir ciertos defectos (Fig. 1).

Presentamos un estudio preliminar de la urografia por RM, empleando tanto secuencias altamente potenciadas en T2 con técnica de adquisición rápida HASTE (URO RM) como aquellas secuencias urográficas por RM por realce de la

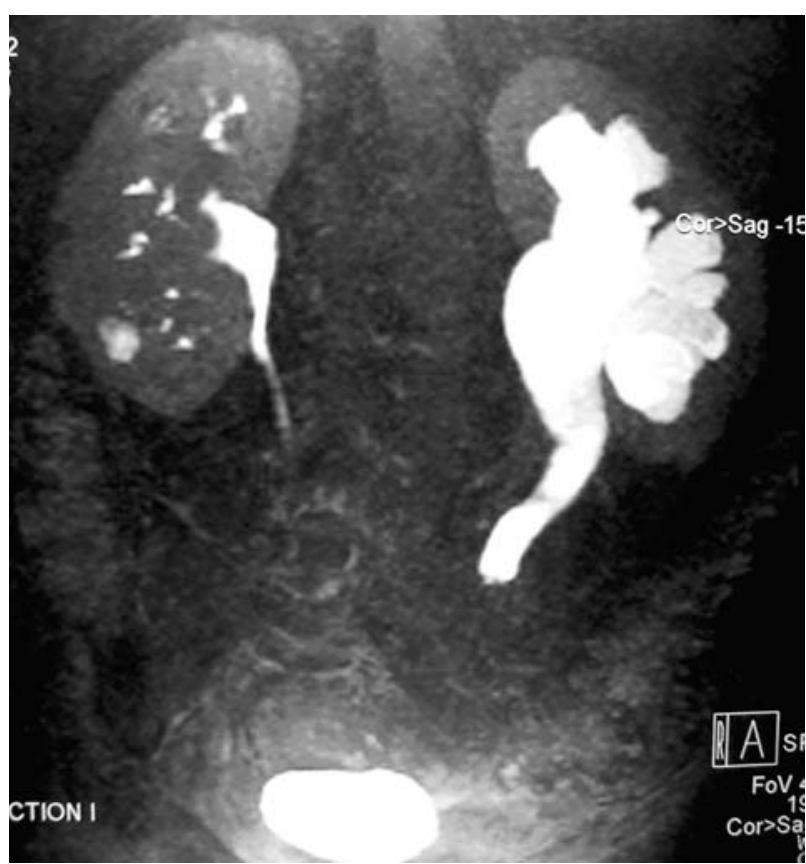

FIGURA 1. Ureterohidronefrosis izquierda causada por tumor ureteral. Corte coronal obtenido tras la reconstrucción de imágenes adquiridas mediante técnica HASTE con proyección de máxima intensidad. 
vía con gadolinio (Gd-URO RM), estableciendo la capacidad diagnóstica para la identificación, así como la localización de la causa responsable del trastorno urológico, y posterior comparación de dichos puntos con la urografía intravenosa. Hemos evaluado, igualmente, la calidad de las imágenes ofrecidas por la urografía por RM en la valoración de cálices, pelvis renal y uréter.

\section{MATERIAL Y MÉTODOS}

\section{Pacientes}

Durante el periodo comprendido entre diciembre de 2002 y julio de 2006, se han realizado en nuestro centro urografias por resonancia magnética en un total de 150 pacientes, 58 mujeres y 92 hombres, con edades comprendidas entre los 12 meses y los 91 años (media 53,35 años). Se realizó estudio con UIV en 63 de los 150 pacientes anteriormente mencionados. Las principales indicaciones para realizar un estudio de urorresonancia fueron la presencia de alergia o antecedentes de reacciones adversas al contraste iónico, en 39 pacientes; el fracaso renal agudo o crónico, en 27; la anulación funcional de alguna de las unidades renales que imposibilita la valoración de la vía urinaria con estudio urográfico convencional, en 18; pacientes en edad pediátrica, 21 casos, a fin de no exponerlos a más radiación ionizante; así como en aquellos casos en los que no pudo alcanzarse el diagnóstico de la patología de base por otras técnicas de imagen, hasta 45 casos (Tabla 1).

De los 150 pacientes estudiados por urorresonancia, en 36 se sospechaba la presencia de una neoplasia de vías, por presentar algún defecto de repleción en el estudio urográfico convencional asociado a hematuria; 21 pacientes en edad pediátrica fueron estudiados por malformaciones

Tabla 1

Causas que motivaron la indicación de urografia por RM.

\begin{tabular}{lc}
\hline Motivo de la indicación & No pacientes \\
\hline Alergia o reacciones adversas & 39 \\
Fracaso renal agudo o crónico & 27 \\
Anulación funcional renal & $18\left(^{*}\right)$ \\
Edad pediátrica & 21 \\
Completar estudio diagnóstico & $45\left(^{*}\right)$ \\
\hline
\end{tabular}

(*) Pacientes con estudio urográfico convencional). en el aparato urinario, evidenciando a lo largo del estudio: 6 ureteroceles, 6 duplicidades pieloureterales y 9 casos con estenosis de la unión pieloureteral; valoramos dos casos de compresión extrínseca ureteral, hallando la causa de obstrucción en ambos, uno por endometriosis y otro por absceso retroperitoneal. 32 pacientes presentaron obstrucción secundaria a litiasis en el tracto urinario superior (TUS) y uno por neoformación vesical. Tres pacientes fueron diagnosticados de tuberculosis genitourinaria y otros tres de pielonefritis aguda de repetición, así como, 18 pacientes con hematuria monosintomática y 39 con ectasia del TUS objetivada por otras técnicas diagnósticas sin constatar patología urológica de base (Tabla 2).

\section{Tabla 2}

Diagnósticos urológicos de los pacientes con estudio por RM

\begin{tabular}{lc}
\hline Patologia de base & No pacientes \\
\hline Tumor de vía superior & $36\left(8^{*}\right)$ \\
Malformaciones ap. Urinario & 21 \\
$\quad$ Ureterocele & 6 \\
$\quad$ Duplicidad pieloureteral & 6 \\
$\quad$ Estenosis unión pieloureteral & 9 \\
Compresión extrinseca ureteral & $2\left(2^{*}\right)$ \\
$\quad$ Endometriosis & 1 \\
$\quad$ Absceso retroperitoneal & 1 \\
Litiasis urinaria & $32\left(32^{*}\right)$ \\
Tumor vesical & 1 \\
Tuberculosis genitourinaria & 3 \\
Pielonefritis aguda de repetición & 3 \\
Hematuria monosintomática & 18 \\
Ectasia del TUS no patológica & $39\left(21^{*}\right)$ \\
\hline
\end{tabular}

$(*)$ Pacientes con estudio urográfico convencional

Contrastamos el resultado del estudio urográfico convencional y por resonancia magnética con los hallazgos anatomopatológicos o quirúrgicos en aquellos pacientes que fueron intervenidos, y con el diagnóstico final al que se llegó por la evolución clínica e imágenes de otras técnicas diagnósticas, en el resto de los casos. 
Urografia intravenosa (UIV)

Tras un ayuno de al menos 5 horas y con una última dieta blanda, realizamos la UIV previa administración de un enema de limpieza intestinal. La técnica, en general, consiste en la realización de una radiografía simple antero-posterior, tras la cual se dispara una serie a los $3,6,12,18$ y 30 minutos tras la inyección de $40-60 \mathrm{~mL}$ de bolo de contraste endovenosos yodado no iónico, como la iopramina (Ultravist 370, Schering(®). El estudio se completa con disparos adicionales o cortes tomográficos, así como placas tardías, hasta pasadas varias horas, según el resultado de las radiografias iniciales.

\section{Urografia por resonancia magnética}

El estudio con urografia por resonancia magnética lo realizamos con una preparación previa del enfermo consistente en ayunas de 10 horas, con última ingesta de una dieta blanda y micción antes de la exploración. Es fundamental conocer previamente la función renal del paciente y realizar una adecuada hidratación, si dicha función lo permite, para el relleno de la vía urinaria, así como la administración, 20 minutos antes de iniciar la prueba, de bajas dosis de furosemida endovenosa en bolo $(0.1 \mathrm{mg} / \mathrm{Kg})$. En la fase de urografía con realce de contraste paramagnético, y unos 5 minutos tras la infusión de la furosemida, administramos gadolinio a razón de 0,1 $\mathrm{mmol} / \mathrm{Kg}$ de peso.

El estudio se realizó con un sistema 1,5 Teslas (T) (Siemens Magneton Sonata), los parámetros del sistema fueron: amplitud de $40 \mathrm{mT} / \mathrm{m}$, velocidad de giro de $200 \mathrm{mT} / \mathrm{m} /$ msec (slew rate) y un "rise time" de $0.3 \mathrm{~ms}$. El protocolo de la exploración se inicia con secuencias bidimensionales localizadoras en Tlw: plano coronal ("true fisp", HASTE con saturación grasa y HASTE sin saturación grasa), plano axial (en $\mathrm{T} 2 \mathrm{w}$ para visualización vesical y renal), planos axial y coronal (GrE Trw, "chemical shift") y plano

\section{Tabla 3} potenciadas en $\mathrm{T} 2 \mathrm{w}$

coronal (3D VIBE). Tras las secuencias localizadoras realizamos secuencias altamente potenciadas en T2w (TSE, ETL=240 echoes; HASTE, con espesor de 60-80, 30-40 y 3-6 mm). Finalmente se completa el estudio con una fase de perfusión, con la administración del gadolinio y la obtención de secuencias urográficas en T1w (120 imágenes Turbo Flash T1w), en planos coronal y axial (3D VIBE). Esta última fase de la exploración sólo se realiza en aquellos pacientes que presentan una función renal normal, una situación de fracaso renal leve o moderado, o una afectación severa de una unidad renal, sin embargo, no se completa en aquellos casos de marcada o severa dilatación pielocalicial asociada a un fracaso renal severo bilateral con atrofia parenquimatosa, realizando exclusivamente las secuencias altamente potenciadas en T2w con detección del fluido estático (Tabla 3).

La calidad de las imágenes en la evaluación de los cálices renales, pelvis renal y uréteres fue valorada siguiendo un baremo con cuatro grados: 0) nula visualización de la vía, sin opacificación de la misma ni distinción de las estructuras; 1) pobre contrastación de la vía con escasa evaluación morfológica, de inferior calidad a la UIV; 2) buena visualización y valoración morfológica de la vía urinaria, con calidad similar o ligeramente inferior a las imágenes ofrecidas por UIV; y 3) muy buena calidad de imágenes, superior a la ofrecida por la UIV, con completa visualización de la vía urinaria y toda su morfologia ${ }^{7}$.

Algoritmo para la combinación de la urografia por RM con secuencias altamente

\begin{tabular}{|c|c|c|}
\hline Función Renal & Estado de la Via Urinaria & Exploración RM \\
\hline Normal & $\begin{array}{l}\text { Normal } \\
\text { Ectasia leve, moderada } \\
\text { o severa unilateral o } \\
\text { bilateral }\end{array}$ & $\begin{array}{l}\text { Urografias por RM-T2w } \\
+ \\
\text { Urografias por RM }-\mathrm{T} 1 \mathrm{w}\end{array}$ \\
\hline $\begin{array}{l}\text { I.R.* leve o moderada } \\
\text { ( }<3 \mathrm{mg} / \mathrm{dL} \text { creatinina) }\end{array}$ & $\begin{array}{l}\text { Normal } \\
\text { Ectasia leve o moderada } \\
\text { unilateral o bilateral } \\
\text { Ectasia severa unilateral. }\end{array}$ & $\begin{array}{l}\text { Urografias por RM }-\mathrm{T} 1 \mathrm{w} \\
+ \\
\text { Urografias por RM-T2w }\end{array}$ \\
\hline $\begin{array}{l}\text { I.R.* severa } \\
\text { (>3 mg/dL creatinina) }\end{array}$ & $\begin{array}{l}\text { Ectasia severa bilateral } \\
\text { Atrofia cortical bilateral. }\end{array}$ & Urografias por RM-T2w \\
\hline
\end{tabular}




\section{Estudio estadístico}

Evaluamos, tanto para la urografía por resonancia magnética como para la convencional, la precisión, la sensibilidad, especificidad, valor predictivo positivo (VPP) y valor predictivo negativo (VPN) en la valoración de la localización de la alteración, como en la capacidad de alcanzar el diagnóstico de la patología de base responsable de las alteraciones morfológicas encontradas en el aparato urinario.

Calculamos la media y la desviación estándar de los valores que obtuvimos en la evaluación de la calidad de las imágenes ofrecidas para la visualización de cálices, pelvis renal y uréter.

\section{RESULTADOS}

La precisión diagnóstica para la localización de la alteración que presentó la urografía por resonancia magnética fue de 83,33\%, mostrando para el mismo fin una sensibilidad del 90\%, una especificidad del 66,66\%, VPP del 87,09\% y VPN del $72,72 \%$. Esta técnica diagnóstica alcanzó una precisión diagnóstica de la patología de base del $83,33 \%$, con una sensibilidad del 89,65\%, especificidad del 69,25\%, VPP del 86,66\% y VPN del $75 \%$.

En los estudios realizados con urografía convencional archivamos una precisión diagnóstica para la localización de la alteración urológica del $85,71 \%$, con sensibilidad del 94,11\%, especificidad del 50\%, VPP del 88,88\% y VPN del 66,66\%. En el diagnóstico de la causa responsable de dichas alteraciones registramos una precisión diagnóstica del 52,38\%, con sensibilidad del 58,82\%, especificidad del 25\%, VPP del $76,92 \%$ y VPN del $12,50 \%$ (Tablas 4 y 5).

En cuanto a la evaluación de la calidad de las imágenes ofrecidas por la urografia por resonancia magnética alcanzamos una media de 2,44 en la evaluación de los cálices renales, de 2,72 en la valoración de la pelvis renal y de 2,12 en la visualización del uréter (Tabla 6).

\section{Tabla 4}

\section{Tabla 5}

\section{DISCUSIÓN}

En el estudio del tracto urinario superior, y especialmente en la valoración de la vía urinaria, la UIV es actualmente la exploración por la imagen de elección, sin embargo, su uso se encuentra limitado principalmente por aquellos casos en los que el paciente presenta reacciones adversas al contraste yodado, alteración de la función renal y en pacientes pediátricos y embarazadas, por la exposición a radiaciones ionizantes no deseables. En todos estos casos la URO RM ofrece la posibilidad de evaluar el aparato urinario y de mostrar la vía urinaria con imágenes de gran calidad sin exponer al paciente a los principales inconvenientes de la $\mathrm{UIV}^{28}, 30$.

El principio de esta técnica esta basado en la adquisición de imágenes altamente potenciadas en T2, lo que genera una baja intensidad de señal de aquellos órganos sólidos y de los fluidos móviles, permitiendo que el fluido estático se realce con una elevada intensidad de señal, lo que afecta al fluido cerebroespinal, al humor acuoso del ojo, al fluido intestinal, a los conductos hepatobiliopancreáticos, a los quistes y al sistema excretor del aparato urinario.

Este realce del fluido estático del aparato urinario en la URO RM, es el que permite el estudio de la vía sin emplear necesariamente un contraste paramagnético, ofreciendo una imagen similar a la de la UIV; además, la técnica HASTE permite evaluar los tejidos circundantes a la vía, aportando información sobre el estado del parénquima renal, incrementando la calidad del estudio morfológico frente a la UIV ${ }^{6}$. Otra de las ventajas

Características de la urografia convencional y de la urografia por RM en el estudio de la localización de la alteración en el aparato urinario

\begin{tabular}{lccccc}
\hline Exploración & Precisión & Sensibilidad & Especificidad & VPP & VPN \\
\hline Urografia IV & $85,71 \%$ & $94,11 \%$ & $50 \%$ & $88,88 \%$ & $66,66 \%$ \\
URO RM & $83,33 \%$ & $90 \%$ & $66,66 \%$ & $87,09 \%$ & $72,72 \%$ \\
\hline
\end{tabular}

Características de la urografía convencional y de la urografía por RM en el estudio de la causa responsable de la alteración en el aparato urinario.

\begin{tabular}{lccccc}
\hline Exploración & Precisión & Sensibilidad & Especificidad & VPP & VPN \\
\hline Urografia IV & $52,38 \%$ & $58,82 \%$ & $25 \%$ & $76,92 \%$ & $12,50 \%$ \\
URO RM & $83,33 \%$ & $89,65 \%$ & $69,25 \%$ & $86,66 \%$ & $75 \%$ \\
\hline
\end{tabular}


Tabla 6

Valor de la media y de la desviación estándar en la evaluación de la calidad de las imágenes urográficas por RM

\begin{tabular}{lccc}
\hline & Cálices & Pelvis & Uréter \\
\hline Media & 2.44 & 2.72 & 2.12 \\
Desv. Estándar & 0.61 & 0.45 & 0.44 \\
\hline
\end{tabular}

que ofrece la URO RM es la información que ofrece el estudio de RM abdominal y pélvica en la fase inicial de la prueba, información que no es capaz de aportar la UIV, lo que podría incluir estudios de extensión de neoplasias, valoración de las causas obstructivas extrínsecas a la vía urinaria y evaluación de alta calidad de la vascularización abdominal, o angiorresonancia, de gran utilidad en vistas a un tratamiento quirúrgico.

Si bien la calidad inicial de las imágenes con URO RM era pobre, el progreso tecnológico, junto con el empleo de diuréticos, ha mejorado notablemente la exploración. El uso de la furosemida, unos 20 a 30 minutos antes de la prueba, permite visualizar mejor la vía urinaria, gracias a que genera una ectasia artificial, lo que resulta especialmente útil en la valoración de toda la longitud ureteral. En la Gd-URO RM, el diurético provoca una dilución homogénea del contraste paramagnético en la vía urinaria, de gran utilidad para el estudio de los cálices, además, es la combinación de gadolinio y furosemida la forma mas eficaz de combatir el efecto $\mathrm{T}^{8}{ }^{8}$, especialmente en los pacientes sin obstrucción o con moderada obstrucción urinaria ${ }^{9}$. Dicho efecto se caracteriza por la aparición de zonas hipointensas a nivel pielocalicial debidas, posiblemente, a un defecto de dilución del gadolinio en la vía.

La administración de altas dosis de furosemida (0.3 $\mathrm{mg} / \mathrm{Kg}$ de peso) ha sido la norma en los inicios de la URO $\mathrm{RM}^{10}{ }^{12}$; si bien, actualmente está más extendido el uso de bajas dosis, es decir, unos $0.05-0.1 \mathrm{mg} / \mathrm{Kg}$ de peso corporal $^{13}$; ya que con dosis superiores a $10 \mathrm{mg}$. el paciente puede requerir la suspensión de la prueba por repleción vesical extrema, aproximadamente a los 30 minutos de la infusión del diurético ${ }^{17}$, y además, se optimiza la calidad de las secuencias T1 en la GdURO RM junto con las dosis estándar de gadolinio (0.1 mmol/Kg de peso corporal) administradas 5 minutos después de la furosemida.
Teóricamente, la administración oral de agua o de suero salino intravenoso puede ser una alternativa capaz de sustituir al diurético ${ }^{18}$, pero resultan menos controlables y consiguen una peor dilución del contraste que la furosemida a bajas dosis ${ }^{17}$.

Combinar las secuencias altamente potenciadas en T2, que ofrecen el estudio del fluido estático en la vía urinaria, y de las $\mathrm{T} 1$ con realce de gadolinio; que alcanza una definición superior de dicha vía, especialmente de los cálices, permite obtener el máximo rendimiento a la RM en el campo de la urología. Dicha combinación está especialmente indicada en pacientes con una función renal normal, o con afectación leve o moderada de ambas unidades renales, o bien, con afectación de leve a severa de una sola unidad renal con normofunción del contralateral; ya que siempre deben determinarse niveles de creatinina sérica no superiores a $2 \mathrm{mg} / \mathrm{dL}$, a fin de mantener la baja nefrotoxicidad y la elevada tolerancia del gadolinio, compuesto de bajo peso molecular y fácil filtración glomerular ${ }^{17}$. Sin embargo, autores como Wille et al. optan por limitar el empleo del gadolinio en el paciente pediátrico a aquellos casos que precisan diferenciar una obstrucción urinaria significativa de una no significativa, con la finalidad de poder indicar un tratamiento quirúrgico resolutivo; así como también en aquellos pacientes donde se requiera de un estudio parenquimatoso renal y no sólo de la vía urinaria ${ }^{15}$ (Fig. 2).

Estudios previos registran una sensibilidad en el diagnóstico de obstrucción del tracto urinario de la urografía por RM del $100 \%$, tal es el caso de Louca et al. ${ }^{19}$, Hussain et $\mathrm{al}^{20}$, O'Malley et $\mathrm{al}^{21} \mathrm{y}$ Regan et al. ${ }^{22,}$ si bien discrepan en la capacidad de esta exploración en la detección de la patología de base. Este diagnóstico se consigue en la mitad de los pacientes de Louca y Hussain; O'Malley et al. presentan una sensibilidad en el diagnóstico etiológico del 90\% y Regan et al. del $100 \%$. Por otro lado, Reuter et al. ${ }^{4}$ registraron una sensibilidad en el diagnóstico de obstrucción y de la causa de base del $96 \%{ }^{23}$. En nuestro estudio se alcanzó una sensibilidad en el diagnóstico de obstrucción urinaria del 90\%, con sensibilidad en el diagnóstico etiológico del 89,65\%. La UIV mostró una sensibilidad similar en el diagnóstico 


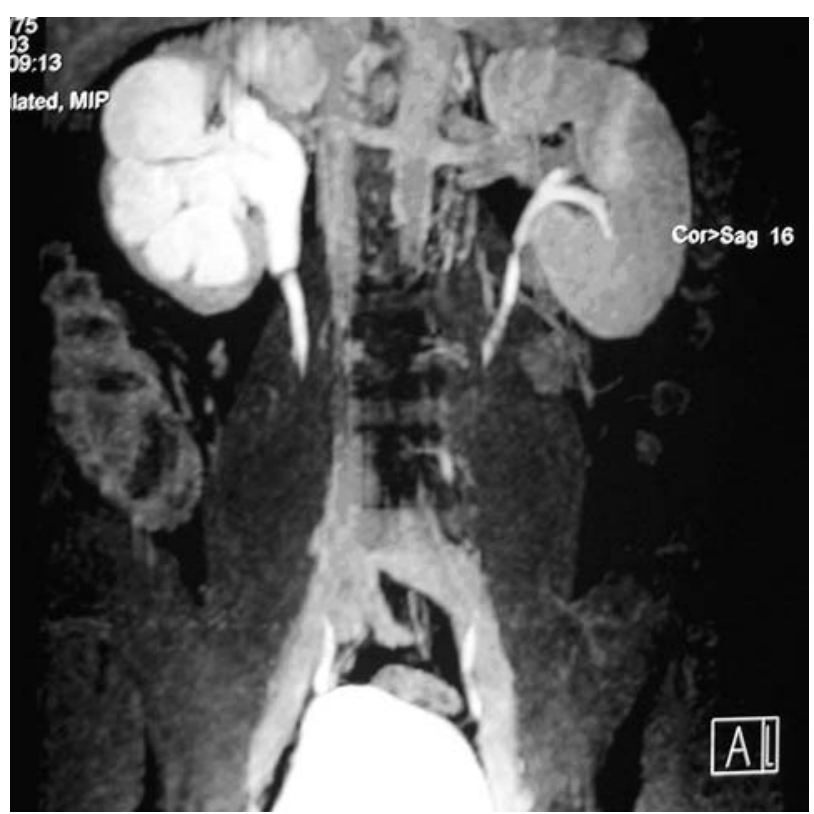

FIGURA 2. Corte coronal de Urorresonancia con administración de Gadolinio en el que se aprecia dilatación pielocalicial secundaria a cruce vascular.

de obstrucción (94,11\%), sin embargo, su sensibilidad en la detección de la patología de base fue notablemente inferior a la registrada por la URO RM $(58,82)$. Las obstrucciones pueden ser dinámicamente visualizadas y cuantificadas con la administración de gadolinio y furosemida, pudiendo recoger y registrar los cambios de la intensidad de señal en la región de interés, (por ejemplo en la pelvis en los casos de obstrucción de la unión pieloureteral). Son estudios similares a los obtenidos con el empleo de radionúclidos en Medicina Nuclear, presentando una gráfica de renograma con dos fases, parenquimatosa y excretora, de similar interpretación a la convencional $^{24}$, pero que aportan imágenes morfológicas renales y de la vía excretora urinaria de mejor calidad, todo ello, sin requerir de radiación ionizante $^{25}$.

En cuanto a la calidad de las imágenes, la pelvis renal es la estructura que mejor consigue visualizar la urografia por RM, alcanzando una calidad casi muy buena $(2,72)$ y superior a la ofrecida por la UIV. La calidad de las imágenes en la evaluación de los cálices alcanza un valor de 2.44 , de buena a muy buena, calidad que se alcanza gracias a la ectasia artificial que la furosemida permite y a la administración del con- traste. Los uréteres presentan la más baja tasa de calidad en la imagen ${ }^{2,12}$, manteniéndose como buena, pero no supera a la UIV, y en algunos casos incluso muestra peor definición de la imagen, a pesar de la administración de la furosemida, que permite apreciar casi toda la longitud ureteral, la interposición del fluido intestinal y vesical son los responsables de la baja percepción de algunos tramos ureterales, aun empleando múltiples proyecciones. Farres et al. presentan un estudio de evaluación de la calidad de la imagen con RM con un baremo similar, consiguiendo para los cálices renales y para pelvis renal un resultado muy similar al nuestro $(2,45$ para cálices y 2,82 para pelvis), registrando una calidad de imagen para uréteres ligeramente superior $(2,55)^{7}$ Hughes et al. describen con un baremo similar la calidad de las imágenes de las diferentes porciones de la vía urinaria en cuatro secuencias diferentes de la prueba, y registran una calidad de imagen para cálices ligeramente superior (entre 1,72 y 2,58), pero algo inferior para pelvis renal (entre 1,72 y 2,55 ) y para uréter (entre 1,47 y 1,88 ) con respecto a nuestra serie ${ }^{26}$.

La detección de litiasis en el aparato urinario puede ser limitada con el uso de la urografia por RM. Su localización a nivel parenquimatoso es muy difícil, mientras que en la vía urinaria al quedar rodeado por el fluido estático o por el gadolinio, se aprecian como defectos de señal de contorno bien definido, hallazgo poco específico que hace difícil su diagnóstico diferencial con los coágulos de sangre, con los clips quirúrgicos y con los pequeños tumores de la vía urinaria ${ }^{8}$, pero también, con falsos defectos de repleción ureteral, o artefactos de la prueba, que se asocian a ectasia de la vía proximal, y se aprecian en los cortes axiales, son de pequeño tamaño y generalmente centrales, posiblemente secundarios a las turbulencias de un flujo urinario más rápido en la porción central ${ }^{13}$. En el terreno de los cálculos urinarios puede tener más relevancia la TAC espiral sin contraste que, aunque exponiendo a radiación ionizante, consigue el diagnóstico de litiasis tanto obstructivas como no obstructivas, y presenta actualmente menor coste. En el diagnóstico diferencial de los defectos en la vía, debe tenerse en cuenta que los cálculos urinarios no muestran señal alguna en ninguna de las 
secuencias de la RM, la sangre se presenta en muchos casos hiperintensa en las secuencias $\mathrm{T} 1$ e hipointensa en las T2, mientras que los tumores de la vía aparecen como engrosamientos de la pared que ocasionalmente pueden realzarse con la administración de gadolinio, debido a su vascularización ${ }^{14}$. En el diagnóstico de litiasis urinaria, Jung et al. consiguen una sensibilidad del $90 \%$ frente al 68\% que alcanza la $\mathrm{UIV}^{23}$, mientras que Sudah et al. archivan con la Gd-URO RM una sensibilidad y especificidad en la detección de cálculos del 100\%, pero registran una sensibilidad menor del 60\% con la URO RM ${ }^{27}$ (Fig. 3).

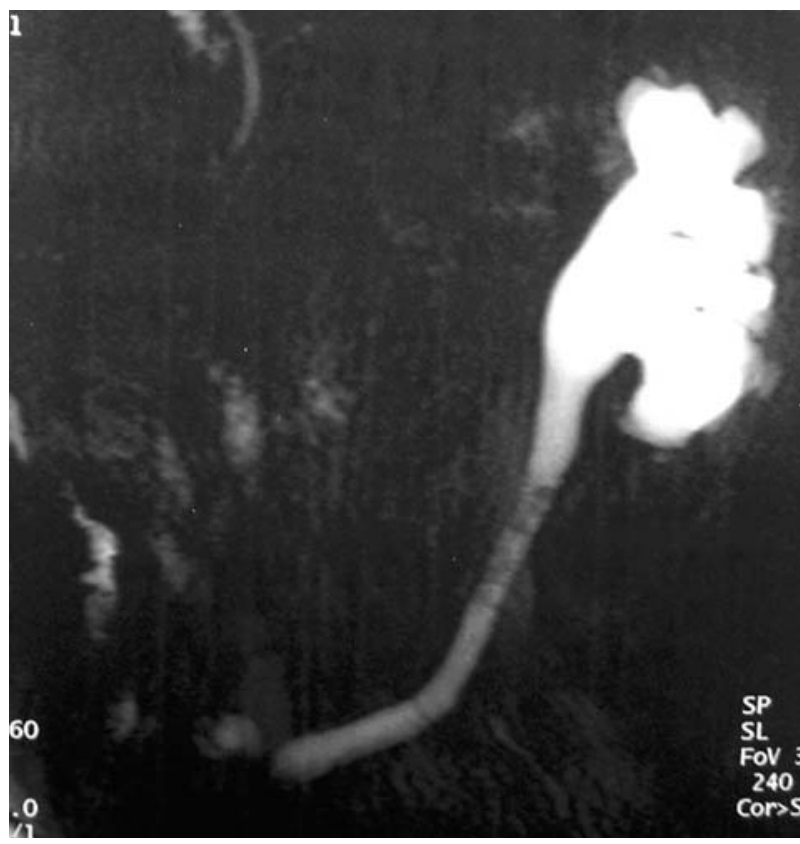

FIGURA 3. Imagen altamente ponderada en T2 en la que se identifican múltiples defectos de replección a nivel ureteral izquierdo correspondientes a neoformación de vias.

Otro de los inconvenientes de la RM es la dificultad que muestra a la hora de diferenciar entre estructuras hiperintensas superpuestas, por ejemplo, resulta frecuente confundir los quistes simples adyacentes a la vía urinaria, con los divertículos caliciales. Al quedar los quistes simples superpuestos a la vía, puede hacernos creer que mantienen una comunicación con la misma. La administración de gadolinio en los casos dudosos permitirá diferenciar qué estructuras mantienen comunicación ${ }^{24}$.
A diferencia de la técnica HASTE, las imágenes obtenidas por RARE están frecuentemente artefactadas por el contenido intestinal, artefacto que no es capaz de eliminar la preparación previa del intestino y que se podría combatir con la ingesta de contraste oral negativo ${ }^{28}$. En nuestra serie el fluido intestinal no ha supuesto una limitación a la exploración gracias a la posibilidad de obtener múltiples proyecciones de la imagen, quedando más dificultada la apreciación de la porción distal ureteral por la interposición del fluido vesical en los pacientes con vejiga a repleción, siendo aconsejable, en caso de que se sospeche patología a este nivel realizar la exploración de esta porción con la vejiga vacía

Otras limitaciones de la RM dependen del paciente, tal es el caso de los pacientes de edad muy avanzada y niños de corta edad que no puedan cooperar, precisando ser sedados en muchos $\operatorname{casos}^{29}$, así como pacientes portadores de implantes metálicos, embarazadas en el primer trimestre y portadores de marcapasos, en los que está contraindicada esta exploración.

\section{CONCLUSIÓN}

La urografía por RM es en la actualidad una exploración que se emplea de forma alternativa a la UIV y a la TAC, especialmente en aquellos pacientes con antecedentes de alergia al contraste yodado, fracaso renal o que no pueden ser expuestos a radiaciones ionizantes, sin embargo, ofrece una mayor capacidad diagnóstica en la patología de aparato urinario, especialmente frente a la UIV, permitiendo un estudio completo, morfológico y funcional, pero también, vascular y de los órganos circundantes.

Teniendo en cuenta que en la obtención habitual del diagnóstico se emplean múltiples técnicas por la imagen, lo que a su vez incrementa la estancia hospitalaria, el coste de la urografia por RM, que permite una evaluación global del aparato urinario, no resulta tan elevado. Además, con el desarrollo de los nuevos hardwares y softwares, no sólo la adquisición de imágenes es más rápida, lo que permite realizar más exploraciones y, por tanto, abaratarlas, además se alcanza una calidad de buena a muy buena, con la prácticamente total visualización de la vía urinaria, similar o superior, en la mayoría de casos, a las imágenes ofrecidas por la UIV. 
Sus escasas limitaciones, especialmente en el terreno de las litiasis urinarias, serán sin duda superadas por los continuos avances tecnológicos, que sumados al progresivo abaratamiento de la exploración pueden convertirla en un plazo medio a corto de tiempo en la técnica por la imagen de elección en el estudio del aparato urinario.

\section{REFERENCIAS}

1. Saxton HM. Review article: urography. $\mathrm{Br} J$ Radiol 1969;42(497):321-346.

2. Hennig J, Nauerth A, Friedburg H. RARE imaging: a fast imaging method for clinical MR. Magn Reson Med 1986:3(6):823-833.

3. Rothpearl A, Frager D, Subramanian A, Bashist B, Baer J, Kay C et al. MR urography: technique and application. Radiology 1995: 194(1):125-130.

4. Reuther G, Kiefer B, Wandl E. Visualization of urinary tract dilatation: value of single-shot MR urography. Eur Radiol 1997: 7(8):1276-1281.

5. Hennig J, Friedburg H. Clinical applications and methodological developments of the RARE technique. Magn Reson Imaging 1988:6(4):391-395.

6. Roy C, Saussine C, Jacqmin D. Magnetic resonance urography. BJU Int 2000:86 Suppl 1:42-47.

7. Farres MT, Gattegno B, Ronco P, Flahault A, Paula-Souza R, Bigot JM. Nonnephrotoxic, dynamic, contrast enhanced magnetic resonance urography: use in nephrology and urology. J Urol 2000:163(4):1191-1196.

8. El Diasty T, Mansour O, Farouk A. Diuretic contrastenhanced magnetic resonance urography versus intravenous urography for depiction of nondilated urinary tracts. Abdom Imaging 2003:28(1): 135-145.

9. Nolte-Ernsting CCA. MR urography examination techniques and clinical applications. Eur Radiol 2001:11:335-377.

10. Hattery RR, King BF. Technique and application of MR urography. Radiology 1995: 194(1):25-27.

11. Di Girolamo M, Pirillo S, Laghi A, Iannicelli E, Fini D, Amadei $\mathrm{M}$ et al. [Urography with magnetic resonance: a new method for the study of the renal collecting system in patients without obstructive uropathy]. Radiol Med (Torino) 1996:92(6):758-764.

12. Nolte-Ernsting CCA. Comparison of gadolinium-enhanced T1-weighted excretory MR urography and static fluid T2weighted MR urography. Eur Radiol 2000:10(suppl):212.

13. Girish G, Chooi WK, Morcos SK. Filling defect artefacts in magnetic resonance urography. Eur Radiol 2004:14(1): 145-150.

14. Blandino A, Gaeta M, Minutoli F, Salamone I, Magno C, Scribano E et al. MR urography of the ureter. AJR Am J Roentgenol 2002: 179(5):1307-1314.

15. Wille S, von Knobloch R, Klose KJ, Heidenreich A, Hofmann R. Magnetic resonance urography in pediatric urology. Scand J Urol Nephrol 2003:37(1):16-21.

16. Klein LT, Frager D, Subramanium A, Lowe FC. Use of magnetic resonance urography. Urology 1998:52(4):602-608.

17. Nolte-Ernsting CCA. MR urography today. Abdom Imaging 2003:28:191-209.
18. Szopinski K, Szopinska M, Borowka A, Jakubowski W. Magnetic resonance urography: initial experience of a lowdose Gd-DTPA-enhanced technique. Eur Radiol 2000: 10(7):1158-1164.

19. Louca G, Liberopoulos K, Fidas A, Nikolakopoulou Z, Lykourinas M, Strigaris K. MR urography in the diagnosis of urinary tract obstruction. Eur Urol 1999:35(2):102-108.

20. Hussain S, O'Malley M, Jara H, Sadeghi-Nejad H, Yucel EK. MR urography. Magn Reson Imaging Clin N Am 1997: 5(1):95-106

21. O'Malley ME, Soto JA, Yucel EK, Hussain S. MR urography: evaluation of a three-dimensional fast spin-echo technique in patients with hydronephrosis. AJR Am J Roentgenol 1997:168(2):387-392.

22. Regan F, Bohlman ME, Khazan R, Rodriguez R, SchultzeHaakh H. MR urography using HASTE imaging in the assessment of ureteric obstruction. AJR Am J Roentgenol 1996: 167(5): 1115-1120.

23. Jung P, Brauers A, Nolte-Ernsting CA, Jakse G, Gunther RW. Magnetic resonance urography enhanced by gadolinium and diuretics: a comparison with conventional urography in diagnosing the cause of ureteric obstruction. BJU Int 2000:86(9):960-965.

24. Borthne A, Nordshus T, Reiseter T, Geitung JT, Gjesdal KI, Babovic A et al. MR urography: the future gold standard in paediatric urogenital imaging? Pediatr Radiol 1999:29(9): 694-701.

25. Teh HS, Ang ES, Wong WC, Tan SB, Tan AG, Chng SM et al. MR renography using a dynamic gradient-echo sequence and low-dose gadopentetate dimeglumine as an alternative to radionuclide renography. AJR Am J Roentgenol 2003: 181(2):441-450.

26. Hughes J, Jan W, Goodie J, Lund R, Rankin S. MR urography: evaluation of different techniques in non-dilated tracts. Clin Radiol 2002:57(11):989-994.

27. Sudah M, Vanninen R, Partanen K, Heino A, Vainio P, AlaOpas M. MR urography in evaluation of acute flank pain: T2-weighted sequences and gadolinium-enhanced threedimensional FLASH compared with urography. Fast lowangle shot. AJR Am J Roentgenol 2001:176(1):105-112.

28. Hirohashi S, Hirohashi R, Uchida H, Kitano S, Ono W, Ohishi $\mathrm{H}$ et al. MR cholangiopancreatography and MR urography: improved enhancement with a negative oral contrast agent. Radiology 1997:203(1):281-285.

29. Kocaoglu M, Ahmet Turan Ilıca, Nail Bulakbası, Atilla Ergin, Bahri Üstünsöz, Tuba Sanal et al. MR urography in pediatric uropathies with dilated urinary tracts. Diagn Interv Radiol 2005; 11:225-232.

30. Garcia-Valtuille R, Garcia-Valtuille AI, Abascal F, Cerezal L, Arguello MC. Magnetic resonance urography: a pictorial overview. Br J Radiol. 2006 Jul;79(943):614-26. Review.

Información autor: Dra. M. Ramos de Campos

Servicio de Urología. Consorcio Hospital General Universitario de Valencia. Avda. Tres Cruces, s/n. 46014 Valencia

E-mail autor: macarenaramos@hotmail.com

Información artículo: Original - Técnicas de imagen

Trabajo recibido: octubre 2006

Trabajo aceptado: diciembre 2006 